\title{
Relaciones de Presiones Óptimas de los Ciclos Complejos de las Turbinas de Gas
}

Raúl Lugo ${ }^{1}$, Juan M. Zamora ${ }^{1}$, Martín Salazar ${ }^{2}$ y Miguel Toledo ${ }^{3}$

(1) Universidad Autónoma Metropolitana-Iztapalapa, Depto. de Ing. de Proc. e Hidráulica, Avda. San Rafael Atlixco No. 186, Col. Vicentina, Iztapalapa, 09340, México, D.F., México (e-mail: lulr@xanum.uam.mx)

(2) Tecnológico de Estudios Superiores de Ecatepec, Div. de Ingeniería Mecatrónica e Industrial, Avda. Tecnológico esquina con Avda. Hank González, Col. Valle de Anáhuac, 55210,

Ecatepec de Morelos, Estado de México, México (e-mail: msalazar@tese.edu.mx)

(3) Instituto Politécnico Nacional, Escuela Superior de Ingeniería Mecánica y Eléctrica, Lab. de Ingeniería Térmica e Hidráulica Aplicada, U.P. Adolfo López Mateos, Edif. 5, $3^{\text {er }}$ piso,

SEPI-ESIME, Col. Lindavista, 07738, México, D.F., México

\begin{abstract}
Resumen
El objetivo de este trabajo es estudiar el comportamiento termodinámico de turbinas de gas complejas que incluyen varias etapas de compresión y enfriamiento, seguidas de dos o más secciones de combustión-expansión. Se obtienen los modelos matemáticos para determinar el trabajo, la eficiencia térmica y sus respectivas relaciones de presiones óptimas en función de las temperaturas de los gases a la entrada de la turbina y del aire a la entrada del compresor, de las eficiencias isentrópicas de compresión y de expansión y del número de etapas de compresión $\mathrm{N}_{c} \mathrm{y}$ expansión $\mathrm{N}_{\mathrm{T}}$. Se presentan curvas paramétricas donde se muestran las relaciones de presiones óptimas para obtener el trabajo y la eficiencia térmica máxima, para cada uno de los casos analizados. Para las turbinas de gas complejas, las relaciones de presiones óptimas se encuentran en un rango aceptable, entre 8.1 y 23.1 para el trabajo máximo y de 17.4 a 32.2 para la eficiencia térmica máxima.
\end{abstract}

Palabras clave: turbina de gas, eficiencia térmica, relación de presiones, ciclos complejos

\section{Optimum Pressure Ratio for Complex Gas Turbine Cycles}

\begin{abstract}
The objective of this work is the characterization of the performance of complex gas turbine cycles that include several compressions with intercooling stages, followed by two or more combustionexpansion sections. Explicit relationships are presented to compute the power output, the thermal efficiency and the optimum pressure ratio values as functions of the gas temperatures at the entrance of the turbine and of the air at the entrance of the compressor, of the isentropic compression and expansion efficiencies, and the number of compression and expansion stages, $\mathrm{N}_{\mathrm{C}}$ and $\mathrm{N}_{\mathrm{T}}$. Parametric curves showing the optimum pressure ratio to obtain maximum power and maximum thermal are presented for each of the cases studied in this work. For complex turbines, the optimum pressure ratios are in an acceptable range, between 8.1 and 23.1 for the maximum power and between 17.4 and 32.2 for the maximum thermal efficiency.
\end{abstract}

Keywords: gas turbine, thermal efficiency, pressure ratio, complex cycles 


\section{INTRODUCCIÓN}

Desde la aparición de las primeras turbinas de gas, se presentaron dos principales problemas a resolver, el primero era obtener la mayor temperatura al inicio de la expansión y el segundo, operar con la máxima eficiencia del compresor y de la turbina. En la actualidad, los avances tecnológicos en el campo de la ingeniería de materiales y el conocimiento de la aerodinámica han contribuido al mejor funcionamiento de las turbinas de gas (Frost et al., 1997). Así mismo, la búsqueda de alternativas para disminuir el trabajo de compresión conduce a aproximar un proceso de compresión a temperatura constante, utilizando dos o más compresores con enfriadores de tipo mecánico, evaporativo, etc. (Bassily, 2004; Fujii et al., 2001). Chen et al. (2004), realizan el estudio de dos compresores con interenfriamiento variando la relación de presiones de compresión y por consiguiente la relación de presiones de enfriamiento, con el objetivo de suministrar el mínimo trabajo de compresión y mejorar la eficiencia térmica.

Yang et al. (2006) y Nishida et al. (2005), realizan otro tipo de propuesta en el análisis termodinámico de las unidades de turbinas de gas, al establecer la configuración de intercambiadores de calor a la salida o entrada del compresor con precalentamiento de aire por medio de la inyección de agua o vapor, para obtener un mayor trabajo motor y eficiencia térmica, manipulando la temperatura del flujo de aire antes de entrar a la cámara de combustión. Otra de las alternativas para obtener mayor trabajo, es la configuración de los ciclos complejos de turbinas de gas, que consisten en compresiones con enfriamiento intermedio y expansiones con calentamiento intermedio, aunque su implementación está sujeta a limitantes tecnológicas (Gazziano, 2007; NATO-OTAN, 2007). Wang et al. (2008), plantean que el análisis de los ciclos complejos puede establecer nuevas configuraciones en los ciclos combinados y en complejos petroleros para generar más potencia con una mayor eficiencia, así mismo aprovechar el aire y los gases de combustión para atemperar corrientes.

La compresión con enfriamiento intermedio permite obtener una aproximación de una compresión isotérmica. Sí la compresión se realiza con tres etapas, entonces se requieren dos ínter-enfriadores; asimismo, la expansión se puede realizar en tres etapas, es decir, con una postcombustión (cámara de combustión) entre las turbinas (Fig. 1). Al generalizar, para $\mathrm{N}_{\mathrm{C}}$ etapas de compresión se utilizan $\left(\mathrm{N}_{\mathrm{C}}-1\right)$ enfriadores intermedios y también se pueden tener $\mathrm{N}_{\mathrm{T}}$ etapas de expansión.

En este trabajo se obtienen los modelos matemáticos del trabajo motor, de la eficiencia térmica y de las relaciones de presiones óptimas para obtener el trabajo motor máximo y la eficiencia térmica máxima, en un ciclo complejo de turbina de gas con $\mathrm{N}_{\mathrm{C}}$ etapas de compresión y con $\mathrm{N}_{\mathrm{T}}$ etapas de expansión. Estos modelos matemáticos se expresan en función de la relación de presiones total, $\pi$, la relación entre la temperatura de los gases a la entrada de la turbina y la del aire a la entrada del compresor, $\boldsymbol{y}$, y de las eficiencias isoentrópicas de compresión, $\eta_{\mathrm{c}}$, y de expansión, $\eta_{\mathrm{t}}$. Los modelos matemáticos se obtienen para dos casos: Caso 1. Cuando las relaciones de presiones de compresión y las relaciones de presiones de expansión son iguales entre si. Caso 2. Cuando las relaciones de presiones de compresión y las relaciones de presiones de expansión son diferentes entre si.

El objetivo del estudio es determinar cuales son los parámetros óptimos del ciclo complejo de una turbina de gas que conducen al trabajo motor máximo o a la eficiencia térmica máxima. En este estudio se hacen las siguientes consideraciones, la composición química, el flujo másico y el calor específico a presión constante del fluido permanece constante. También se desprecian las pérdidas de carga en las cámaras de combustión y en los enfriadores.

\section{DESARROLLO DEL MODELO DE LA TURBINA DE GAS CON TRES ETAPAS DE COMPRESIÓN Y TRES ETAPAS DE EXPANSIÓN}

En una turbina de gas con enfriamiento intermedio entre los compresores de alta, media y baja presión; la temperatura del aire a la entrada de los compresores de presión intermedia y de alta presión es igual a la temperatura del aire a la entrada del compresor de baja presión; además esta configuración cuenta con postcombustión intermedia entre las turbinas, que permite elevar la 
temperatura de los gases a la misma que tenían a la entrada de la turbina de alta presión. En la Fig. 1 se muestra el diagrama esquemático de la turbina de gas que se estudia en esta sección.

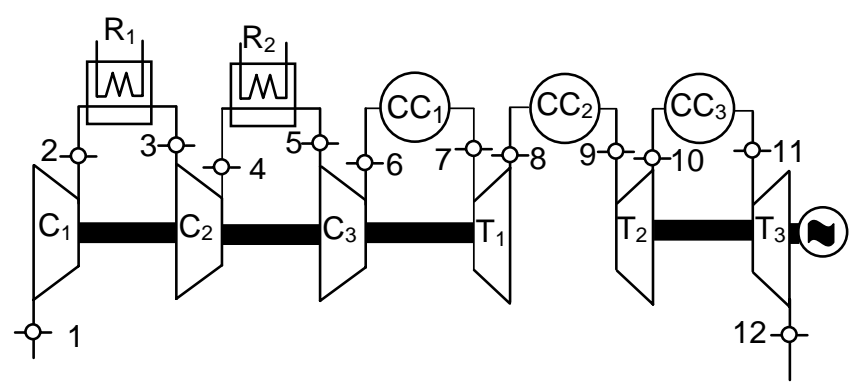

Fig. 1: Turbina de gas con tres etapas de compresión y tres etapas de expansión.

El ciclo termodinámico descrito por el fluido se representa en el diagrama temperatura entropía de la Fig. 2, este ciclo se compone de los siguientes procesos: compresiones politrópicas, (1-2), (3-4), (56); calentamientos a presión constante, (6-7), (8-9), (10-11); expansiones politrópicas, (7-8), (9-10), (11-12); enfriamientos isobáricos, (2-3), (4-5) y (12-1).

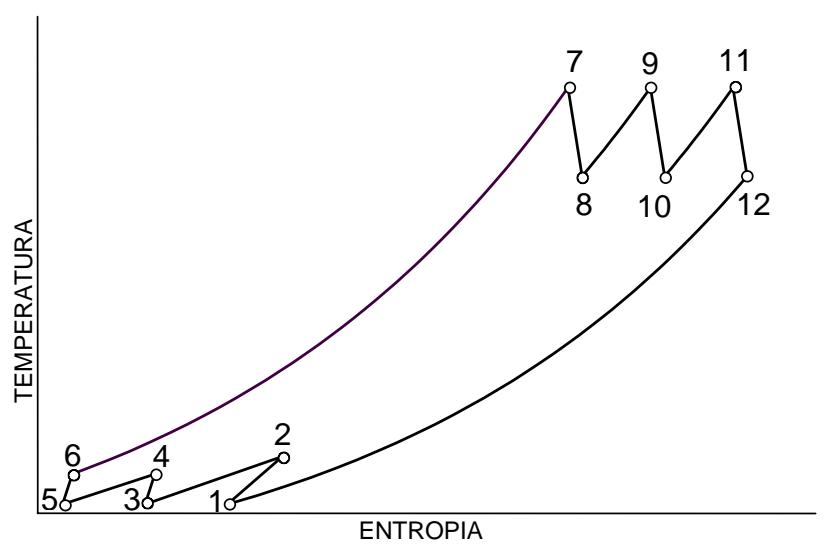

Fig. 2: Diagrama temperatura entropía.

Trabajo motor

El trabajo motor en función de y, $\eta_{\mathrm{c}}, \eta_{\mathrm{t}}, \pi_{\mathrm{C} 1}, \pi_{\mathrm{C} 2}, \pi_{\mathrm{C} 3}, \pi_{\mathrm{T} 1}, \pi_{\mathrm{T} 2} \mathrm{y} \pi_{\mathrm{T} 3}$ se expresa de la siguiente manera.

$\mathrm{w}_{\mathrm{M}} / \mathrm{C}_{\mathrm{P}} \mathrm{T}_{1}=\mathrm{y} \eta_{t}\left(3-1 / \pi_{T 1}^{x}-1 / \pi_{T 2}^{x}-1 / \pi_{T 3}^{x}\right)-1 / \eta_{c}\left(\pi_{C 1}^{x}+\pi_{C 2}^{x}+\pi_{C 3}^{x}-3\right)$

en donde

$y=T_{7} / T_{1}=T_{9} / T_{1}=T_{11} / T_{1} \quad X=R / C_{P}$.

La relación de presiones de cada etapa de compresión y de expansión se liga a la relación de presiones total, $\pi$, de la siguiente manera:

$\pi=\pi_{C 1} \pi_{C 2} \pi_{C 3}=\pi_{T 1} \pi_{T 2} \pi_{T 3}$.

El trabajo motor contiene la suma de las relaciones $\pi_{\mathrm{C} 1}, \pi_{\mathrm{C} 2}$ y $\pi_{\mathrm{C} 3}$ que tienen el mismo exponente, ec. (1); y para que la suma de estas tres relaciones de presiones sea mínima, éstas deben ser iguales. Entonces, la relación de presiones de cada una de las tres etapas de compresión es:

$\pi_{C 1}=\pi_{C 2}=\pi_{C 3}=\pi^{1 / 3}$. 
De manera análoga se obtienen las relaciones de presiones de expansión $\pi_{\mathrm{T}_{1}}, \pi_{\mathrm{T} 2}$ y $\pi_{\mathrm{T} 3}$ :

$\pi_{T 1}=\pi_{T 2}=\pi_{T 3}=\pi^{1 / 3}$.

Entonces, el trabajo motor en función de $\pi, y, \eta_{c} y \eta_{t}$ se expresa de la siguiente manera:

$w_{M} / C_{P} T_{1}=3 y \eta_{t}\left(1-1 / \pi^{x / 3}\right)-3 / \eta_{c}\left(\pi^{x / 3}-1\right)$.

Es evidente que el aumento de $y, \eta_{c} y$ de $\eta_{t}$ ejercen una influencia favorable sobre el trabajo motor.

La relación de presiones óptima, $\pi_{\mathrm{A}}$, para obtener el trabajo motor máximo, es la siguiente:

$\pi_{\mathrm{A}}^{\mathrm{x} / 3+\mathrm{x} / 3}=\mathrm{y} \eta_{c} \eta_{t}$

Calor suministrado

El calor suministrado al ciclo de la turbina de gas se expresa como sigue:

$\mathrm{q}_{\mathrm{A}} / \mathrm{C}_{\mathrm{p}} \mathrm{T}_{1}=\mathrm{y}-1+\mathrm{y} \eta_{t}\left(2-1 / \pi_{T 1}^{x}-1 / \pi_{T 2}^{x}\right)-1 / \eta_{c}\left(\pi_{c 3}^{x}-1\right)$

Caso 1. Las relaciones de presiones de expansión y las relaciones de presiones de compresión son iguales entre si

La expresión del calor suministrado en función de la relación de presiones total, $\pi$, es la siguiente;

$q_{A} / C_{P} T_{1}=y-1+2 y \eta_{t}\left(1-1 / \pi^{x / 3}\right)-1 / \eta_{c}\left(\pi^{x / 3}-1\right)$.

Caso 2. Las relaciones de presiones de expansión y las relaciones de presiones de compresión son diferentes entre si

En la ec. (8), se encuentra la suma $\pi_{\mathrm{T} 1}$ y $\pi_{\mathrm{T} 2}$ con el mismo exponente, esta suma es máxima cuando:

$$
\pi_{T 1}=\pi_{\mathrm{T} 2}=\left(\pi / \pi_{\mathrm{T} 3}\right)^{1 / 2},
$$

entonces, el calor suministrado en función de $\pi, \pi_{\mathrm{T} 3}, \mathrm{y} \pi_{\mathrm{C} 3}$ es:

$q_{A} / C_{P} T_{1}=y-1+2 y \eta_{t}\left(1-\pi_{T 3}^{x / 2} / \pi^{x / 2}\right)-1 / \eta_{c}\left(\pi_{C 3}^{x}-1\right)$.

\section{Eficiencia térmica}

El modelo matemático para calcular la eficiencia térmica se obtiene para dos casos.

Caso 1. Las relaciones de presiones de expansión y las relaciones de presiones de compresión son iguales entre si.

$$
\eta_{T H}=\frac{3 y \eta_{t}\left(1-\frac{1}{\pi^{x / 3}}\right)-\frac{3}{\eta_{c}}\left(\pi^{x / 3}-1\right)}{y-1+2 y \eta_{t}\left(1-\frac{1}{\pi^{x / 3}}\right)-\frac{1}{\eta_{c}}\left(\pi^{x / 3}-1\right)} .
$$

La relación de presiones óptima, $\pi_{\mathrm{B}}$, es: 
$\pi_{B}^{X / 3+X / 3}=y \eta_{c} \eta_{t}(3 / 3)\left[\left(3-2 \eta_{T H}\right) /\left(3-\eta_{T H}\right)\right]$.

Caso 2. Las relaciones de presiones de expansión y las relaciones de presiones de compresión son diferentes entre si

La eficiencia térmica queda en función de $y, \eta_{\mathrm{c}}, \eta_{\mathrm{t}}, \pi_{\mathrm{C} 1}, \pi_{\mathrm{C} 2}, \pi_{\mathrm{C} 3}, \pi_{\mathrm{T} 1}, \pi_{\mathrm{T} 2} \mathrm{y} \pi_{\mathrm{T} 3}$ :

$\eta_{T H}=1-\frac{y-1-y \eta_{t}\left(1-\frac{1}{\pi_{T 3}^{x}}\right)+\frac{1}{\eta_{c}}\left(\pi_{C 1}^{x}+\pi_{C 2}^{x}-2\right)}{y-1-\frac{1}{\eta_{c}}\left(\pi_{C 3}^{x}-1\right)+y \eta_{t}\left(1-\frac{1}{\pi_{T 1}^{x}}\right)+y \eta_{t}\left(1-\frac{1}{\pi_{T 2}^{x}}\right)}$

sustituyendo las ecs. (4) y (5) en la ec. (14), se obtiene la expresión de la eficiencia térmica en función de $\pi, \pi_{\mathrm{T} 3}, \mathrm{y} \pi_{\mathrm{C} 3}$ :

$\eta_{T H}=1-\frac{y-1-y \eta_{t}\left(1-\frac{1}{\pi_{T 3}^{x}}\right)+\frac{2}{\eta_{c}}\left(\frac{\pi^{x / 2}}{\pi_{C 3}^{x / 2}}-1\right)}{y-1-\frac{1}{\eta_{c}}\left(\pi_{C 3}^{x}-1\right)+2 y \eta_{t}\left(1-\frac{\pi_{T 3}^{x / 2}}{\pi^{x / 2}}\right)}$.

La relación de presiones $\pi_{\mathrm{C} 3}$ óptima es la siguiente:

$\pi_{C 3}=\pi^{1 / 3} /\left(1-\eta_{T H}\right)^{2 / 3 X}$.

y la relación de presiones $\pi_{\mathrm{T} 3}$ óptima es:

$\pi_{T 3}=\pi^{1 / 3} /\left(1-\eta_{T H}\right)^{2 / 3 x}$,

entonces, las relaciones de presiones $\pi_{\mathrm{T} 1}$ y $\pi_{\mathrm{T} 2}$ valen:

$\pi_{T 1}=\pi_{T 2}=\left(1-\eta_{T H}\right)^{1 / 3 x} \pi^{1 / 3}$.

La relación entre las relaciones de presiones $\pi_{\mathrm{T} 3} \operatorname{con} \pi_{\mathrm{T} 1}$ y $\pi_{\mathrm{T} 2}$ es:

$\pi_{T 3} / \pi_{T 1}=\pi_{T 3} / \pi_{T 2}=1 /\left(1-\eta_{T H}\right)^{1 / X}$.

La ec. (19) muestra que la relación de presiones de la última etapa de la turbina es mayor que las precedentes.

La expresión de la eficiencia térmica en función sólo de $\pi$ es:

$$
\eta_{T H}=1-\frac{y-1-y \eta_{t}\left[1-\frac{\left(1-\eta_{T H}\right)^{2 / 3}}{\pi^{x / 3}}\right]+\frac{2}{\eta_{c}}\left(\pi^{x / 3}\left(1-\eta_{T H}\right)^{1 / 3}-1\right)}{y-1-\frac{1}{\eta_{c}}\left[\frac{\pi^{x / 3}}{\left(1-\eta_{T H}\right)^{2 / 3}}-1\right]+2 y \eta_{t}\left(1-\frac{1}{\pi^{x / 3}\left(1-\eta_{T H}\right)^{1 / 3}}\right)}
$$

Finalmente, la relación de presiones óptima, $\pi_{\mathrm{B}}$, para obtener la eficiencia térmica máxima vale: 
$\pi_{B}^{X / 3+x / 3}=y \eta_{c} \eta_{t}\left(1-\eta_{T H}\right)^{1 / 3}$

Para determinar los valores de $\pi_{\mathrm{B}}$ y de $\eta_{\mathrm{TH}}$ se hace de manera iterativa, partiendo de una estimación de $\eta_{\mathrm{TH}}$ y utilizando alternativamente para el caso 1 las ecs. (12) y (13) y para el caso 2 utilizando alternativamente las ecs. (20) y (21) hasta la convergencia.

\section{DESARROLLO DEL MODELO DE LA TURBINA DE GAS CON TRES ETAPAS DE COMPRESIÓN Y DOS ETAPAS DE EXPANSIÓN}

En la Fig. 3 se muestra el diagrama esquemático de la turbina de gas que se estudia en esta sección. El ciclo termodinámico descrito por el fluido se representa en el diagrama temperatura entropía de la Fig. 4.

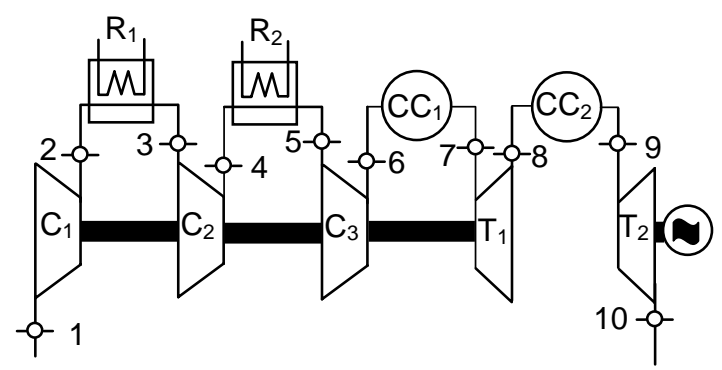

Fig. 3: Turbina de gas con tres etapas de compresión y dos etapas de expansión.

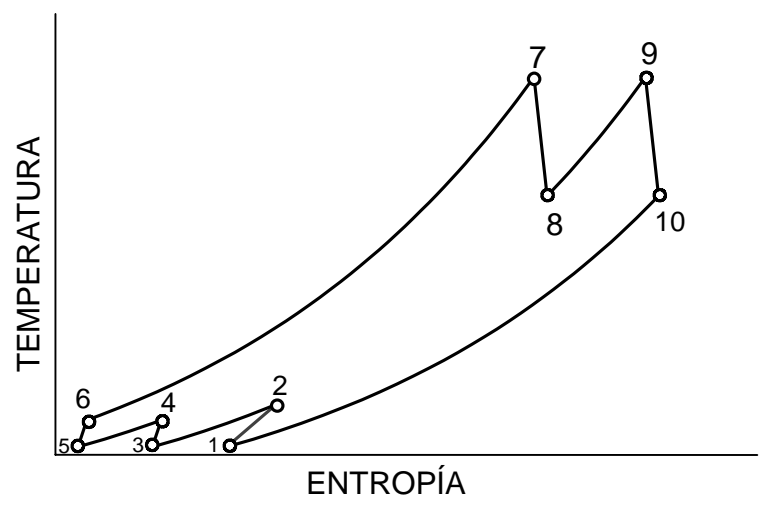

Fig. 4: Diagrama temperatura entropía.

Trabajo motor

La expresión del trabajo motor es:

$w_{M} / C_{P} T_{1}=y \eta_{t}\left(2-1 / \pi_{T 1}^{x}-1 / \pi_{T 2}^{x}\right)-1 / \eta_{c}\left(\pi_{C 1}^{x}+\pi_{C 2}^{x}+\pi_{C 3}^{x}-3\right)$.

En la ec. (22), se encuentra la suma de $\pi_{\mathrm{T}_{1}}$ y $\pi_{\mathrm{T} 2}$ elevados al mismo exponente; esta suma será máxima cuando:

$\pi_{T 1}=\pi_{T 2}=\pi^{1 / 2}$

entonces, el trabajo motor en función sólo de $\pi$ es:

$w_{M} / C_{P} T_{1}=2 y \eta_{t}\left(1-1 / \pi^{x / 2}\right)-3 / \eta_{c}\left(\pi^{x / 3}-1\right)$.

y la relación de presiones óptima, $\pi_{\mathrm{A}}$, es:

$\pi_{A}^{X / 3+x / 2}=y \eta_{c} \eta_{t}$. 
El calor suministrado se expresa de la siguiente manera

$q_{A} / C_{P} T_{1}=y-1+y \eta_{t}\left(1-1 / \pi_{T 1}^{x}\right)-1 / \eta_{c}\left(\pi_{C 3}^{x}-1\right)$

se muestra, que el calor suministrado es función de $\pi_{\mathrm{T} 1}$ y $\pi_{\mathrm{C} 3}$.

Caso 1. Las relaciones de presiones de expansión y las relaciones de presiones de compresión son iguales entre si

$q_{A} / C_{P} T_{1}=y-1+y \eta_{t}\left(1-1 / \pi^{x / 2}\right)-1 / \eta_{c}\left(\pi^{x / 3}-1\right)$.

Caso 2. Las relaciones de presiones de expansión y las relaciones de presiones de compresión son diferentes entre si

La relación de presiones $\pi_{\mathrm{T} 1}$ se expresa como sigue

$\pi_{\mathrm{T} 1}=\pi / \pi_{\mathrm{T} 2}$,

entonces, el calor suministrado en función de $\pi, \pi_{\mathrm{T} 2}, \mathrm{y} \pi_{\mathrm{C} 3}$ vale:

$q_{A} / C_{P} T_{1}=y-1+y \eta_{t}\left(1-\pi_{T 2}^{x} / \pi^{x}\right)-1 / \eta_{c}\left(\pi_{C 3}^{x}-1\right)$.

Eficiencia térmica

Caso 1. Las relaciones de presiones de expansión y las relaciones de presiones de compresión son iguales entre si

$\eta_{T H}=\frac{2 y \eta_{t}\left(1-\frac{1}{\pi^{x / 2}}\right)-\frac{3}{\eta_{c}}\left(\pi^{x / 3}-1\right)}{y-1+y \eta_{t}\left(1-\frac{1}{\pi^{x / 2}}\right)-\frac{1}{\eta_{c}}\left(\pi^{x / 3}-1\right)}$.

y la relación de presiones óptima, $\pi_{\mathrm{B}}$, para obtener la eficiencia térmica máxima:

$\pi_{B}^{X / 3+X / 2}=y \eta_{c} \eta_{t}(3 / 2)\left(\left[2-\eta_{T H}\right] /\left[3-\eta_{T H}\right]\right)$.

Caso 2. Las relaciones de presiones de expansión y las relaciones presiones de compresión son diferentes entre si.

$\eta_{T H}=1-\frac{y-1-y \eta_{t}\left(1-\frac{1}{\pi_{T 2}^{x}}\right)+\frac{2}{\eta_{c}}\left(\frac{\pi^{x / 2}}{\pi_{C 3}^{X / 2}}-1\right)}{y-1-\frac{1}{\eta_{c}}\left(\pi_{C 3}^{x}-1\right)+y \quad \eta_{t}\left(1-\frac{\pi_{T 2}^{x}}{\pi^{x}}\right)}$.

la relación de presiones óptima $\pi_{\mathrm{C} 3}$ está expresada en la ec. (16), y la relación de presiones óptima $\pi_{\mathrm{T} 2}$ es igual a:

$\pi_{T 2}=\pi^{1 / 2} /\left(1-\eta_{T H}\right)^{1 / 2 X}$.

La relación de presiones $\pi_{\mathrm{T} 1}$ es la siguiente: 
$\pi_{T 1}=\pi^{1 / 2}\left(1-\eta_{T H}\right)^{1 / 2 X}$

La relación entre $\pi_{\mathrm{T} 2} \mathrm{y} \pi_{\mathrm{T} 1}$ da la siguiente expresión

$$
\pi_{T 2} / \pi_{T 1}=1 /\left(1-\eta_{T H}\right)^{1 / X}
$$

Se observa que la relación de presiones de la última etapa de la turbina es mayor que las precedentes.

Finalmente, la eficiencia térmica en función sólo de $\pi$ es:

$$
\eta_{T H}=1-\frac{y-1-y \eta_{t}\left[1-\frac{\left(1-\eta_{T H}\right)^{1 / 2}}{\pi^{x / 2}}\right]+\frac{2}{\eta_{c}}\left(\pi^{x / 3}\left(1-\eta_{T H}\right)^{1 / 3}-1\right)}{y-1-\frac{1}{\eta_{c}}\left[\frac{\pi^{x / 3}}{\left(1-\eta_{T H}\right)^{2 / 3}}-1\right]+y \eta_{t}\left(1-\frac{1}{\pi^{x / 2}\left(1-\eta_{T H}\right)^{1 / 2}}\right)}
$$

La relación de presiones óptima, $\pi_{\mathrm{B}}$, para obtener la eficiencia térmica máxima se expresa de la siguiente manera

$$
\pi_{B}^{X / 3+X / 2}=y \eta_{c} \eta_{t}\left(1-\eta_{T H}\right)^{1 / 6} .
$$

\section{DESARROLLO DEL MODELO DE LA TURBINA DE GAS CON N $\mathrm{N}_{\mathrm{T}}$ ETAPAS DE EXPANSIÓN}

En esta sección se generaliza la metodología desarrollada en las secciones anteriores para una turbina de gas con $\mathrm{N}_{\mathrm{C}}$ etapas de compresión y $\mathrm{N}_{\mathrm{T}}$ etapas de expansión.

El trabajo motor para $N_{C}$ etapas de compresión y $N_{T}$ etapas de expansión se expresa de la siguiente manera

$w_{M} / C_{P} T_{1}=N_{T} y \eta_{t}\left(1-1 / \pi^{x / N_{T}}\right)-N_{C} / \eta_{c}\left(\pi^{x / N_{C}}-1\right)$.

La relación de presiones óptima para obtener el trabajo motor máximo para $\mathrm{N}_{\mathrm{C}}$ etapas de compresión y $\mathrm{N}_{\mathrm{T}}$ etapas de expansión es:

$\pi_{A}^{X / N_{C}+X / N_{T}}=y \eta_{c} \eta_{t}$.

\section{Calor suministrado}

Caso 1. Las relaciones de presiones de expansión y las relaciones de presiones de compresión son iguales entre si

$q_{A} / C_{P} T_{1}=y-1+\left(N_{T}-1\right) y \eta_{t}\left(1-1 / \pi^{x / N_{T}}\right)-1 / \eta_{c}\left(\pi^{x / N_{C}}-1\right)$.

Caso 2. Las relaciones de presiones de expansión y las relaciones presiones de compresión son diferentes entre si

$q_{A} / C_{P} T_{1}=y-1+\left(N_{T}-1\right) y \eta_{t}\left(1-\pi_{T N_{T}}^{X /\left(N_{T}-1\right)} / \pi^{X /\left(N_{T}-1\right)}\right)-1 / \eta_{c}\left(\pi_{C_{N C}}^{X / N_{C}}-1\right)$ 


\section{Eficiencia térmica}

Caso 1. Las relaciones de presiones de expansión y las relaciones de presiones de compresión son iguales entre si

$$
\eta_{T H}=\frac{N_{T} y \eta_{t}\left(1-\frac{1}{\pi^{x / N_{T}}}\right)-\frac{N_{C}}{\eta_{c}}\left(\pi^{x / N_{C}}-1\right)}{y-1+\left(N_{T}-1\right) y \eta_{t}\left(1-\frac{1}{\pi^{X / N_{T}}}\right)-\frac{1}{\eta_{c}}\left(\pi^{x / N_{C}}-1\right)} .
$$

La relación de presiones óptima, $\pi_{\mathrm{B}}$, para obtener la eficiencia térmica máxima se expresa de la siguiente manera

$$
\pi_{B}^{X / N_{C}+X / N_{T}}=y \eta_{C} \eta_{t}\left(N_{C} / N_{T}\right)\left(\left[N_{T}-\eta_{T H}\left(N_{T}-1\right)\right] /\left[N_{C}-\eta_{T H}\right]\right) .
$$

Caso 2. Las relaciones de presiones de expansión y las relaciones de presión de compresión son diferentes entre si

$$
\left(1-\eta_{T H}\right)=\frac{y-1-y \eta_{t}\left[1-\frac{\left(1-\eta_{T H}\right)^{\left(N_{T}-1\right) / N_{T}}}{\pi^{X / N_{T}}}\right]+\frac{N_{C}-1}{\eta_{C}}\left(\pi^{x / N_{C}}\left(1-\eta_{T H}\right)^{1 / N_{C}}-1\right)}{y-1+\left[N_{T}-1\right] y \eta_{t}\left(1-\frac{1}{\pi^{X / N_{T}}\left(1-\eta_{T H}\right)^{1 / N_{T}}}\right)-\frac{1}{\eta_{C}}\left[\frac{\pi^{x / N_{C}}}{\left(1-\eta_{T H}\right)^{\left(N_{C}-1\right) / N_{C}}-1}\right]}
$$

La relación de presiones óptima, $\pi_{\mathrm{B}}$, para obtener la eficiencia térmica máximas es:

$$
\pi_{B}^{x / N_{C}+x / N_{T}}=y \eta_{c} \eta_{t}\left(1-\eta_{T H}\right)^{1-1 / N_{C}-1 / N_{T}} .
$$

Haciendo la relación entre $\pi_{B}$ y $\pi_{A}$ se encuentra la siguiente expresión:

$$
\left(\pi_{B} / \pi_{A}\right)^{X / N_{C}+X / N_{T}}=\left(1-\eta_{T H}\right)^{1-1 / N_{C}-1 / N_{T}} .
$$

Se comprueba que según el número de etapas de compresión y expansión, el valor óptimo de $\pi_{B}$ puede ser mayor, igual o menor que $\pi_{\mathrm{A}}$. En particular para $\mathrm{N}_{\mathrm{C}}=\mathrm{N}_{\mathrm{T}}=2$, se tiene que $\pi_{\mathrm{B}}=\pi_{\mathrm{A}}$; $\sin$ embargo, los ciclos son diferentes, porque en el caso 1, el compresor y la turbina están divididos en dos etapas con la misma relación de presiones y generan el mismo trabajo motor; en el caso 2, la segunda etapa tiene una relación de presiones mayor y efectúa un trabajo motor mayor que la primera etapa.

\section{RESULTADOS Y ANÁLISIS DE RESULTADOS}

En la Fig. 5 se presentan los resultados del análisis numérico de la ec. (38) en función de la relación de presiones, $\pi$, para los siguientes valores: $\mathrm{y}=4.42, \eta_{\mathrm{c}}=0.85, \eta_{\mathrm{t}}=0.88$.

En la Fig. 6 se muestran las eficiencias térmicas en función de la relación de presiones para los valores de $\mathrm{y}=4.42, \eta_{\mathrm{c}}=0.85$ y $\eta_{\mathrm{t}}=0.88$. Se muestra la curva para la turbina de gas simple $\left(\mathrm{N}_{\mathrm{C}}=1\right.$ $\mathrm{N}_{\mathrm{T}}=1$ ) con la finalidad de tomarla como referencia. Se observa que las curvas de los ciclos de las turbinas complejas de gas [ $\left(\mathrm{N}_{\mathrm{C}}=3, \mathrm{~N}_{\mathrm{T}}=3\right) ;\left(\mathrm{N}_{\mathrm{C}}=3, \mathrm{~N}_{\mathrm{T}}=2\right) ;\left(\mathrm{N}_{\mathrm{C}}=2, \mathrm{~N}_{\mathrm{T}}=2\right)$ ] tienen un aspecto plano en la región de su valor máximo; entonces la relación de presiones se puede alejar bastante de la relación de presiones óptima sin perder mucho trabajo motor y eficiencia térmica. Además, se encuentra que en los ciclos complejos óptimos, las relaciones de presiones máximas están entre 25 y 150 bar a 
excepción del arreglo $\mathrm{N}_{\mathrm{T}}=3, \mathrm{~N}_{\mathrm{C}}=3$ que es mayor a 200 bar. Para los arreglos $\left[\left(\mathrm{N}_{\mathrm{C}}=3, \mathrm{~N}_{\mathrm{T}}=1\right) ;\left(\mathrm{N}_{\mathrm{C}}=2\right.\right.$, $\left.N_{T}=1\right) ;\left(N_{T}=1, N_{C}=1\right)$ ], después de la relaciones de presiones óptima, la eficiencia disminuye considerablemente a medida que la relación de presiones se incrementa.

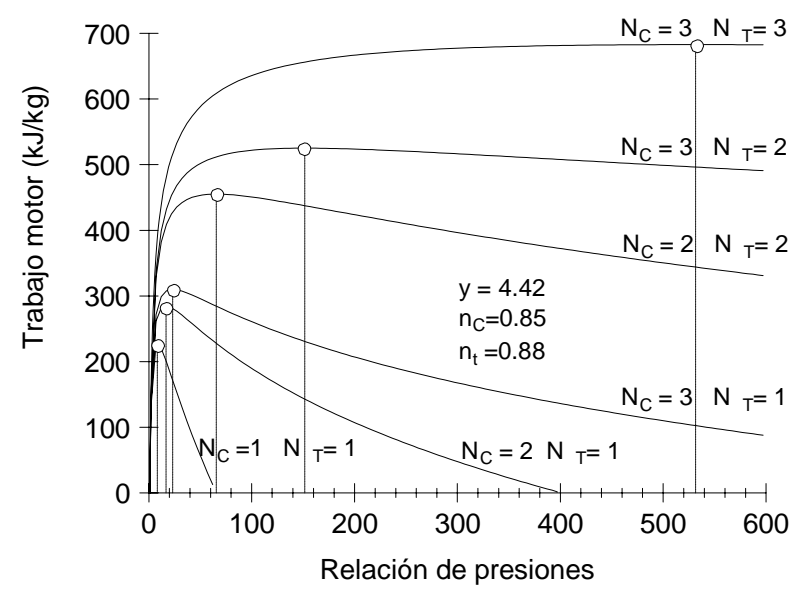

Fig. 5: Trabajo motor en función de la relación de presiones.

En la Tabla 1 se muestra que de acuerdo al número de etapas de compresión y expansión, el valor óptimo de la relación de presiones para obtener la eficiencia térmica máxima, $\pi_{B}$, puede ser mayor, igual o menor que la relación de presiones óptima para obtener el trabajo motor máximo, $\pi_{\mathrm{A}}$. En particular, para $N_{C}=N_{T}=2$, se tiene que las relaciones de presiones para obtener la eficiencia térmica máxima y el trabajo máximo son iguales, es decir, $\pi_{\mathrm{B}}=\pi_{\mathrm{A}}$.

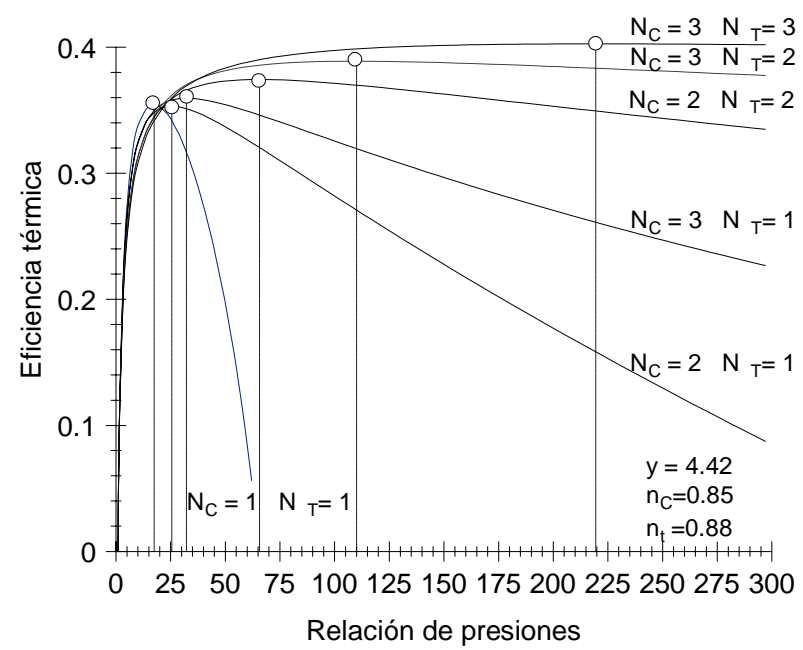

Fig. 6: Eficiencia térmica en función de la relación de presiones.

En la Fig. 7 se muestra que para una turbina compleja con un arreglo de $N_{T}=3$ y $N_{C}=2$, las relaciones de presiones óptimas para obtener los trabajos motores máximos y las eficiencias térmicas máximas aumentan a medida que la relación de temperaturas y aumenta, por ejemplo, para $\mathbf{y}=4.76$ las relaciones de presiones óptimas son $\pi_{\mathrm{A}}=208.16$ y $\pi_{\mathrm{B}}=147.08$ y para y $=5.11$ son $\pi_{\mathrm{A}}=279.66$ y $\pi_{\mathrm{B}}$ $=193.76$, respectivamente. Sin embargo, para este arreglo las relaciones de presiones óptimas para obtener los trabajos motores máximos son mayores que las relaciones de presiones óptimas para obtener las eficiencias térmicas máximas. Por citar un caso, para y $=4.42$, las relaciones de presiones óptimas para obtener el trabajo motor máximo y la eficiencia térmica máxima son 151.52 y 109.47 respectivamente, esto también se muestra en las Tablas 1 y 2. 


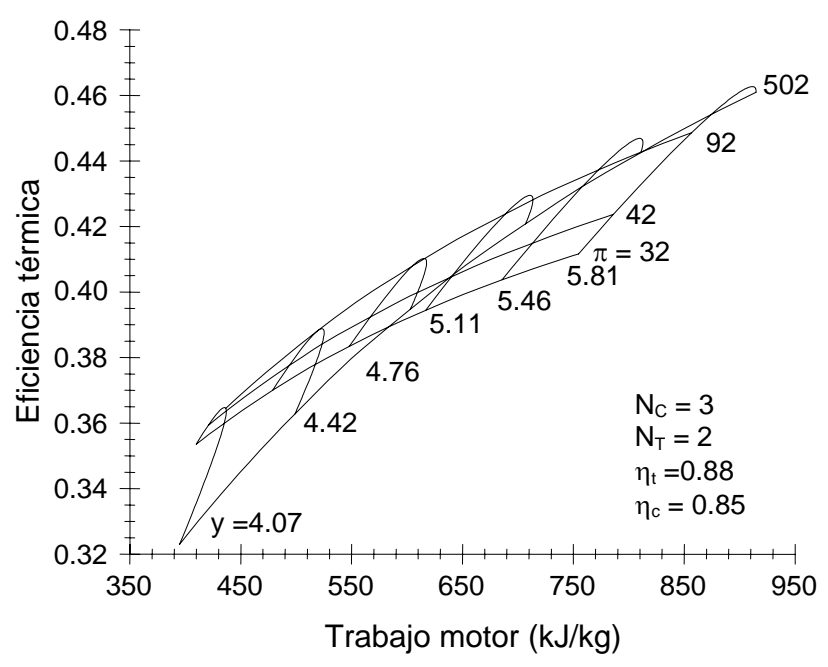

Fig. 7: Eficiencia térmica máxima en función del trabajo motor para diferentes relaciones de presiones y diferentes relaciones de temperaturas.

Tabla 1: Trabajos motores máximos y eficiencias térmicas máximas con sus respectivas relaciones de presiones óptimas.

\begin{tabular}{crrccccc}
\hline $\mathrm{y}=4.42$ & & \multicolumn{1}{c}{$\pi_{\mathrm{A}}$} & $\mathrm{W}_{\max }(\mathrm{kJ} / \mathrm{kg})$ & $\eta_{\pi \mathrm{A}}$ & \multicolumn{1}{l}{$\pi_{\mathrm{B}}$} & $\eta_{\max }$ & $\mathrm{W}_{\pi \mathrm{B}}(\mathrm{kJ} / \mathrm{kg})$ \\
\hline $\mathrm{N}_{\mathrm{C}}=\mathbf{3}$ & $\mathrm{N}_{\mathrm{T}}=3$ & 531.6 & 682.7 & .3965 & 219.5 & .4026 & 669.5 \\
& 2 & 151.5 & 525.3 & .3876 & 109.5 & .3888 & 523.3 \\
& 1 & 23.1 & 310.1 & .3565 & 32.2 & .3594 & 307.3 \\
$\mathbf{2}$ & $\mathbf{2}$ & $\mathbf{6 5 . 6}$ & $\mathbf{4 5 5 . 1}$ &. $\mathbf{3 7 4 3}$ & $\mathbf{6 5 . 6}$ & .3743 & $\mathbf{4 5 5 . 1}$ \\
& 1 & 16.8 & 284.5 & .3461 & 25.6 & .3531 & 278.2 \\
$\mathbf{1}$ & 1 & 8.1 & 227.6 & .3205 & 17.4 & .3540 & 197.7 \\
\hline
\end{tabular}

Tabla 2: Trabajos motores máximos y eficiencias térmicas máximas con sus respectivas relaciones de presiones óptimas para un arreglo de $\mathrm{N}_{\mathrm{T}}=3$ y $\mathrm{N}_{\mathrm{C}}=2$.

\begin{tabular}{cccrl}
\hline $\mathrm{y}$ & $\pi_{\mathrm{A}}$ & $\mathrm{w}_{\max }(\mathrm{kJ} / \mathrm{kg})$ & \multicolumn{1}{c}{$\pi_{\mathrm{B}}$} & $\eta_{\max }$ \\
\hline 4.07 & 107.47 & 437.02 & 79.50 & 0.3647 \\
4.42 & 151.52 & 525.30 & 109.47 & 0.3888 \\
4.76 & 208.16 & 617.66 & 147.08 & 0.4102 \\
5.11 & 279.66 & 713.62 & 193.76 & 0.4294 \\
5.46 & 368.49 & 812.80 & 250.79 & 0.4468 \\
5.81 & 477.35 & 914.88 & 319.38 & 0.4627 \\
\hline
\end{tabular}

En la Fig. 8 se muestra que las relaciones de presiones óptimas aumentan a medida que la relación de temperaturas aumenta; también aumentan a medida que el número de etapas de compresión aumenta para cualquier arreglo de turbinas de gas complejas. Para el arreglo $\left(N_{C}=3 N_{T}=3\right)$, se muestra que el intervalo de las relaciones de presiones óptimas es de 152 a 745 (se incrementa en un 388.73\%), el cual es muy elevado; sin embargo, la eficiencia térmica se incrementa desde 0.37869 hasta 0.47569 , que corresponde a un 25.62 por ciento. Para este arreglo, también se puede ver que la pendiente es menor a medida que la relación de temperaturas aumenta, por ejemplo al aumentar de 4.07 a 4.42 la eficiencia térmica se incrementa en un 6.34 por ciento y al aumentar de 5.46 a 5.81 solamente se incrementa en un 3.39 por ciento. Para los arreglos $\left(N_{C}=3 N_{T}=2\right)$ y $\left(N_{T}=2\right.$ $\mathrm{N}_{\mathrm{C}}=2$ ), el intervalo de las relaciones de presiones óptimas es de 49 a 320; la eficiencia térmica se incrementa en un 26.85 y en un 27.38 por ciento respectivamente; por consiguiente la pendiente es mayor que para el arreglo de $\left(N_{C}=3 N_{T}=3\right)$. Para los arreglos $\left(N_{C}=3 N_{T}=1\right),\left(N_{C}=2 N_{T}=1\right)$ y $\left(N_{C}=1\right.$ $\mathrm{N}_{\mathrm{T}}=1$ ), el intervalo de las relaciones de presiones óptimas es de 14 a 72 , la eficiencia térmica se 
incrementa en un 29.89 y en un 32.37 por ciento respectivamente; por lo tanto, sus pendientes son mayores que para los arreglos de $\left(N_{C}=3 N_{T}=3\right),\left(N_{C}=3 N_{T}=2\right)$ y $\left(N_{T}=2 N_{C}=2\right)$.

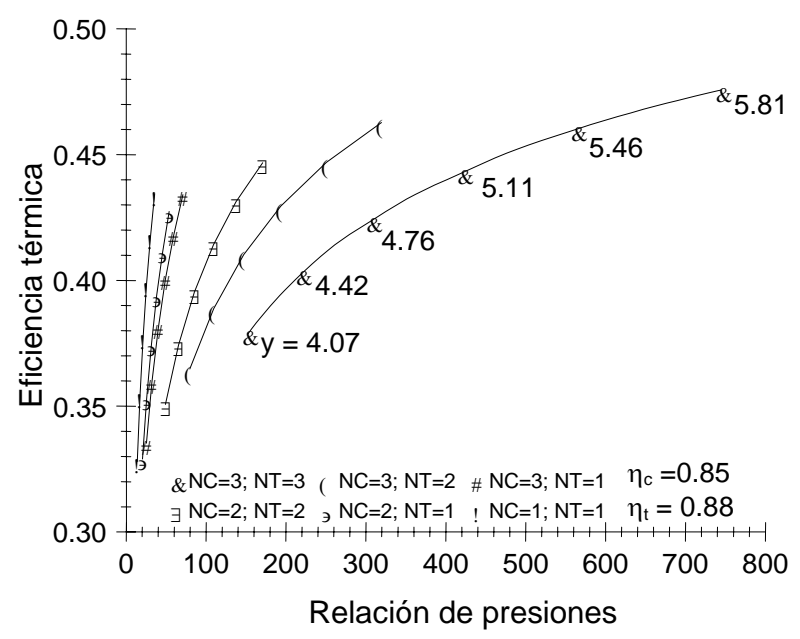

Fig. 8: Eficiencia térmica máxima en función de las relaciones de presiones óptimas a diferentes temperaturas a la entrada de la turbina.

En la Fig. 9 se muestra que para el arreglo de $N_{C}=N_{T}=3$, se obtiene la mayor eficiencia térmica pero con una relación de presiones óptima muy elevada, para cualquier eficiencia isoentrópica de compresión. También, se muestra que a medida que aumenta la eficiencia isoentrópica de compresión, la eficiencia térmica aumenta; y que a medida que la eficiencia isoentrópica de compresión aumenta, las relaciones de presiones óptima para obtener las eficiencias térmicas máximas también aumentan para cualquier arreglo. Las pendientes de las curva de los arreglos $\left(N_{C}=3 N_{T}=3\right),\left(N_{C}=3 N_{T}=2\right),\left(N_{T}=2 N_{C}=2\right),\left(N_{C}=3 N_{T}=1\right)$ y $\left(N_{C}=2 N_{T}=1\right)$ se incrementan en este orden de los arreglos, es decir, la eficiencia térmica se incrementa en un 11.04, 11.98, 12.46, 14.49 y 15.05 por ciento, y la relación de presiones disminuye en 65.26, 57.1, 51.07, 42.89 y 41.11 por ciento, respectivamente. Sin embargo, para el arreglo $\left(\mathrm{N}_{\mathrm{C}}=1 \mathrm{~N}_{\mathrm{T}}=1\right)$ la eficiencia térmica se incrementa en un 17.99 por ciento, y la relación de presiones en un 43.82 por ciento.

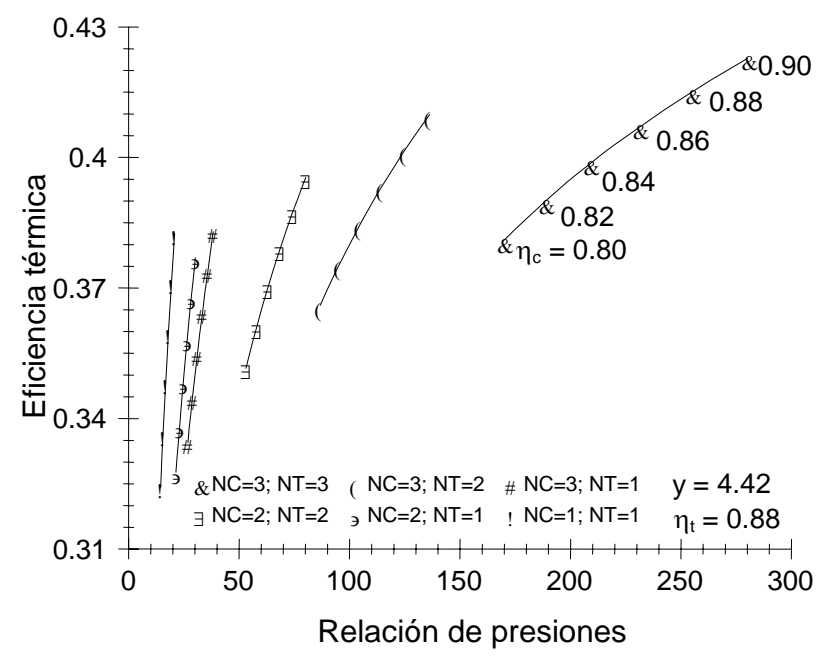

Fig. 9: Eficiencia térmica máxima en función de las relaciones de presiones óptimas a diferentes eficiencias isoentrópicas de compresión.

En la Fig. 10 se muestra que a medida que aumenta la eficiencia isoentrópica de expansión la eficiencia térmica aumenta; y que a medida que la eficiencia isoentrópica de expansión aumenta, las relaciones de presiones óptima para obtener las eficiencias térmicas máximas también aumentan 
para cualquier arreglo. Para los arreglos ( $\left.N_{C}=3 N_{T}=3\right),\left(N_{C}=3 \quad N_{T}=2\right),\left(N_{T}=2 \quad N_{C}=2\right),\left(N_{C}=3 \quad N_{T}=1\right)$ las pendientes de sus respectivas curvas se incrementan en este orden de los arreglos, es decir, se incrementa el porcentaje de la eficiencia térmica en un 13.69, 15.85, 17.03, 22.44 por ciento, y la relación de presiones disminuye ligeramente en un 37.04, 36.71, 36.53 y 36.41 por ciento, respectivamente. Para el arreglo $\left(\mathrm{N}_{\mathrm{C}}=2 \mathrm{~N}_{\mathrm{T}}=1\right)$ la eficiencia térmica máxima se incrementa en 24.02, y la relación de presiones óptima aumenta en un 38.01 por ciento. Sin embargo, para el arreglo $\left(N_{C}=1 N_{T}=1\right)$ la eficiencia térmica máxima se incrementa en un 33.53 por ciento, y la relación de presiones se incrementa en un 60 por ciento.

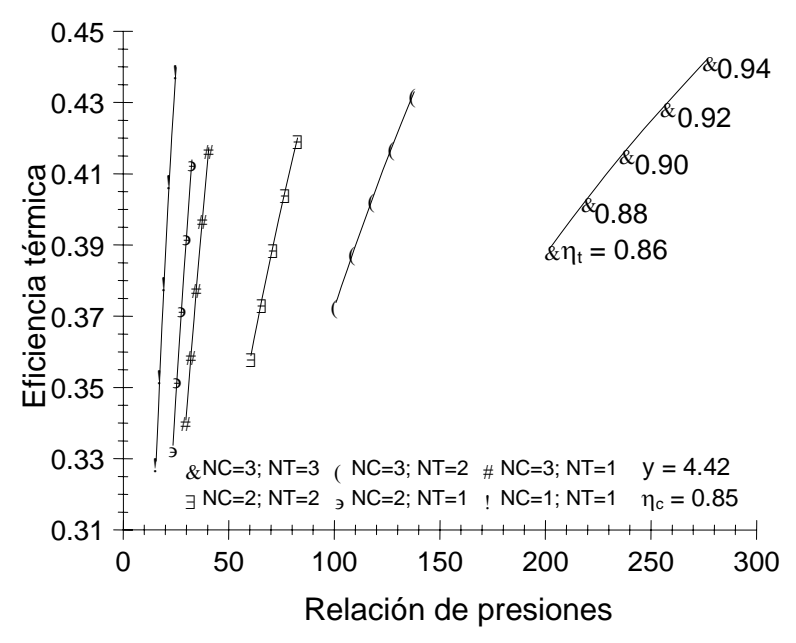

Fig. 10: Eficiencia térmica máxima en función de la relación de presiones óptimas a diferentes eficiencias isoentrópicas de expansión.

En la Fig. 11 se muestra la eficiencia térmica en función de la relación de presiones óptima a diferentes temperaturas a la entrada de las turbinas de gas y para diferentes tipos de arreglos, considerando que las $\eta_{c}$ y $\eta_{t}$ sean iguales a 0.88 . Se muestra que para obtener las eficiencias térmicas máximas se requieren relaciones de presiones óptimas elevadas. Analizando el comportamiento de la eficiencia térmica para una temperatura de $1300{ }^{\circ} \mathrm{C}$, se tiene que a medida que el número de etapas de expansión disminuye la relación de presiones óptima también disminuye.

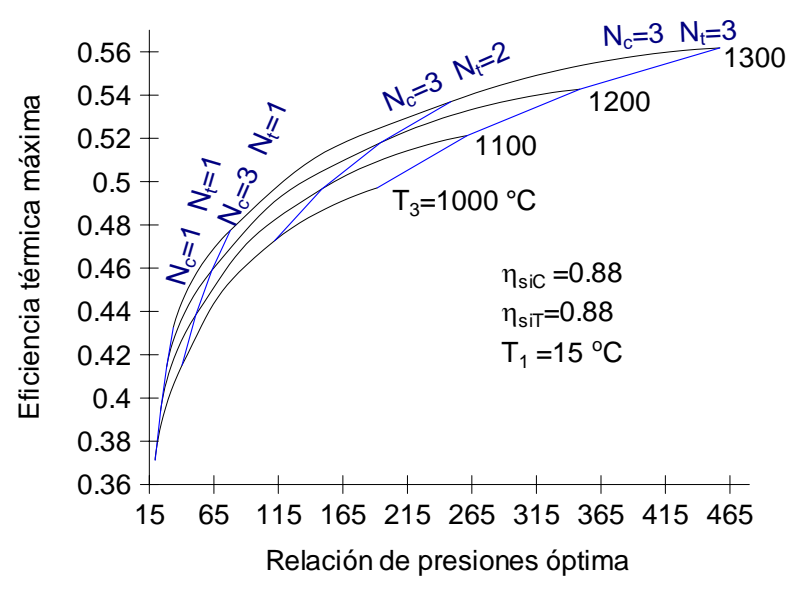

Fig. 11: Eficiencia térmica máxima en función de la relación de presiones óptimas a diferentes temperaturas a la entrada de la turbina.

Al comparar los resultados de las Figs. 6 y 11 se encuentra que las eficiencias térmicas son menores cuando las relaciones de presiones de compresión y expansión son iguales en cada etapa. 


\section{CONCLUSIONES}

Las mayores eficiencias térmicas se obtienen para el caso en que las $\pi_{\mathrm{C}}$ son diferentes y las $\pi_{\mathrm{T}}$ también son diferentes.

Según el número de etapas de compresión y expansión, el valor óptimo de la relación de presiones para obtener la eficiencia térmica máxima, puede ser mayor, igual o menor que la relación de presiones óptima para obtener el trabajo motor máximo. En particular, para $\mathrm{N}_{\mathrm{C}}=\mathrm{N}_{\mathrm{T}}=2$, se tiene que las relaciones de presiones para obtener la eficiencia térmica máxima y el trabajo máximo son iguales.

La eficiencia térmica del ciclo de turbina de gas con una sola etapa de compresión y expansión es del 43.25 por ciento; y para una turbina de gas con tres etapas de compresión y 3 etapas de expansión se alcanzan una eficiencia máxima de 56.18 por ciento; estos valores son para sus respectivas relaciones de presiones óptimas. Sin embargo, la implementación de las tres etapas de compresión y expansión para obtener la máxima eficiencia requiere que la relación de presiones sea 13 veces mayor que la relación de presiones óptima del ciclo simple.

\section{NOMENCLATURA}

$\mathrm{C}_{\mathrm{p}}$ Calor específico a presión constante; [kJ/kgK],

$\mathrm{h}$ entalpía por unidad de masa; [kJ/kg],

$\mathrm{N}$ número de etapas; [-],

q calor por unidad de masa; [kJ/kg],

$\mathrm{R}$ constante particular del aire; [kJ/kg K],

$\mathrm{T}$ temperatura; $\left[{ }^{\circ} \mathrm{C}, \mathrm{K}\right]$,

w trabajo por unidad de masa; [kJ/kg],

$X$ relación entre la constante particular del aire y el calor específico a presión constante; [-],

y relación de temperaturas, definida en la ec. (2); [-],

$\eta$ eficiencia; [-],

$\pi$ relación de presiones; [-].

\section{Subíndices}

A suministrado,

C compresor,

M motor,

c isoentrópico de compresión,

$\mathrm{t}$ isoentrópico de expansión,

T turbina,

TH térmica.

\section{REFERENCIAS}

Bassily A.M.; Performance improvements of the intercooler reheat recuperated gas-turbine cycle using absorption inlet-cooling and evaporative after-cooling, Applied Energy: 77, 249-272 (2004).

Chen L., W. Wang, F. Sun y Ch. Wu; Closed intercooler regenerator Brayton-cycle with constanttemperature heat-reservoirs. Applied Energy: 77, 429-446 (2004).

Frost T.H., A. Anderson y B. Agnew; A hybrid gas turbine cycle (Brayton/Ericsson), Proc. Instn. Mech. Eng. Part A: J. Power Energy: 211, 121-131 (1997). 
Fujii S., K. Kaneko, K. Otani e Y. Tsujikawa; Mirror gas turbine: a newly proposed method of exhaust heat recovery, Trans. ASME J. Eng. Gas Turbine Power: 123, 481-486 (2001).

Gazziano M.; Hot section life Assessment by a creep model to plant gas turbine based power plant electricity production. Thesis comprehensive summary. University of Florencia, Department of mechanical, Italy, (2007). www.efpe.org/DownloadThesis.asp?file=4\&thesis=96.

NATO-OTAN; AC/323(AVT-036)TP/106: Performance Prediction and Simulation of Gas Turbine Engine Operation for Aircraft, Marine, Vehicular, and Power Generation, (2007). http://www.rta.nato.int/pubs/rdp.asp?RDP=RTO-TR-AVT-036.

Nishida K., T. Takagi y S. Kinoshita; Regenerative steam-injection gas-turbine systems, Applied Energy: 81, 231-246 (2005).

Wang J., Y. Dai y L. Gao; Parametric analysis and optimization for a combined power and refrigeration cycle, Applied Energy: 85(11), 1071-1085 (2008).

Yang Y., B. Lin y J. Chen; Influence of regeneration on the performance of a Brayton refrigerationcycle working with an ideal Bose-gas, Applied Energy: 83, 99-112 (2006). 